\title{
Application effects of cadmium and humic acid on the growth, chlorophyll fluorescence, leaf gas exchange and secondary metabolites in misai kucing (Orthosiphon stamineus) benth
}

\begin{abstract}
Aims: This experiment was conducted to investigate and distinguish the relationships in the production of total phenolics, total flavonoids, chlorophyll content, total biomass, leaf area, leaf nitrate, proline, net photosynthesis and chlorophyll fluorescence parameters under three levels of cadmium application $(0,3,6 \mathrm{mg} / \mathrm{kg})$ and three concentration of humic acid (HA) (0, $50,100 \mathrm{mg} / \mathrm{L}$ ) for 12 weeks in Orthosiphon stamineus Benth. Study Design: Stem cuttings of O. staminues were propagated for two weeks in small pots and Original Research Article Ibrahim et al.; ARRB, 18(3): 1-14, 2017; Article no.ARRB.36401 2 then transferred to pots filled with a soilless mixture of burnt rice husk and coco peat (ratio 3:1). Orthosiphon stamineus seedlings were exposed to three levels of $\mathrm{Cd}(0,3$ and $6 \mathrm{mg} / \mathrm{kg}$ in the form of $\mathrm{CdCl}$ 2) during media preparation and three levels of humic acid (HA) $(0,50$ and $100 \mathrm{mg} / \mathrm{L}$ ). Soluble humic acid as potassium-humate (90\% humic acid, 11-13\% K2O) was used. This factorial experiment was organized in a randomized complete block (RCBD) design with three replications. Place and Duration of Study: Methodology: The experiment was performed for 12 weeks using 135 plants. The measurement of photosynthesis was obtained from a closed infra-red gas analyzer LICOR 6400XT Portable Photosynthesis System (IRGA, Licor Inc., USA). Total phenolics and flavonoid was determined using Follin-Ciocalteau reagent, Nitrate using Cardy nitrate meter, Chlorophyll fluorescence was measured using a portable chlorophyll fluorescence meter (Handy PEA, Hansatech Instruments Ltd., Norwich, UK) and Proline was determined by using Glacial acetic acid and ninhydrin reagent. Results: It was found that all parameters except chlorophyll fluorescence parameters were influenced by interaction effects between cadmium and humid acid $(\mathrm{P} \leq 0.05)$. As the concentration of cadmium increased $(0>6 \mathrm{mg} / \mathrm{kg})$ the production of plant total phenolics, flavonoids and proline increased but the production of total biomass, leaf area, net photosynthesis, total chlorophyll content and nitrate uptake was reduced. The application of humid acid can reduce negative effects of cadmium. As humid acid level increases from 0 to $100 \mathrm{mg} / \mathrm{L}$ the negative effects of cadmium on total biomass, leaf area, net photosynthesis and total chlorophyll were decreased. Conclusion: This work reveals that the use of cadmium can enhance the production of secondary metabolites in O. stamineus (total phenolics and flavonoids). The study showed the negative effects of cadmium on plant growth, Gas exchange and chlorophyll fluorescence can be reduced by enhancing application of humic acid. The application of humic acid also was found to reduce the cadmium uptake of this herb. Present study showed that high levels of cadmium can lowered the nitrate concentration in this plant. This showed that application of cadmium and humic acid can serve to be a useful tool to enhance secondary metabolites properties of this plant.
\end{abstract}

Keyword: Medicinal plants; Cadmium; Humic acid; Growth; Biochemical; Leaf gas exchange 